\title{
COMUnicación
}

\section{Fecundidad de M ylossoma duriventre (Palometa) en Ucayali durante el Ciclo Reproductivo 2011-2012}

\author{
Fecundity of Mylossoma duriventre (Silver Mylossoma) in Ucayali during the \\ 2011-2012 ReProduCtIVE CYCLE
}

Javier Zaldívar E. ${ }^{1,2}$, José Carlos Riofrío Q., ${ }^{1,3}$

\section{Resumen}

\begin{abstract}
Mylossoma duriventre «palometa» es un pez de la familia Characidae, explotado comercialmente para consumo humano directo en la región de Ucayali, Perú. El objetivo del estudio fue ampliar los conocimientos reproductivos de la especie estimando su fecundidad. Se recolectaron 49 ejemplares en estadio IV y V de madurez que fueron desembarcados en el puerto de Pucallpa durante el ciclo reproductivo 2011-2012. Se registró la longitud (LT) y peso (PT) total de cada pez, así como el peso de las gonadas (PGón). Se tomaron submuestras de las gónadas para el conteo de los ovocitos y determinación de la fecundidad, aplicando la ecuación de regresión lineal simple $\mathrm{F}=\mathrm{a}+\mathrm{bx}$, donde $\mathrm{F}=$ fecundidad, $\mathrm{x}=$ longitud total, peso total o peso del ovario, $\mathrm{y}$ a $\mathrm{y} \mathrm{b}$ son los coeficientes de la regresión $(\mathrm{a}=$ intercepto, $\mathrm{b}=$ tasa de incremento de la fecundidad con respecto a las variables de análisis). La fecundidad absoluta media se estimó en 68280 ovocitos, que corresponde a una talla media de $21.3 \mathrm{~cm}$ de LT (rango de 10749 a 134665 ovocitos en ejemplares con tallas de 18.5 a $28.0 \mathrm{~cm}$ ). La mejor correlación al analizar las regresiones entre el número de ovocitos con la LT, PT y PGón fue con esta última, siendo la ecuación $\mathrm{N} .{ }^{\circ}$ de ovocitos $=1618.1$ PGon $+11735\left(\mathrm{r}^{2}=0.7917 ; \mathrm{p}<0.05\right)$.
\end{abstract}

Palabras clave: palometa, Mylossoma duriventre, fecundidad, pesquería amazónica, Ucayali

\section{Abstract}

Mylossoma duriventre «Silver Mylossoma» is a fish of the family Characidae, commercially exploited for human consumption in the region of Ucayali, Peru. The aim of the present study was to improve the reproductive knowledge of the species in relation to fecundity. Forty nine specimens were collected from landings in Pucallpa during the

\footnotetext{
${ }^{1}$ Facultad de Ciencias Biológicas, Universidad Nacional Mayor de San Marcos, Lima, Perú

2 († 01.03.2012)

${ }^{3}$ E-mail: jriofrioq@yahoo.com
}

Recibido: 26 de octubre de 2014

Aceptado para publicación: 12 de setiembre de 2015 
2011-2012 reproductive cycle. Measurements of total length (LT), total weight (PT) and the weight of gonads (PGon) of each fish were recorded. Subsamples of gonads were taken for oocyte counting to determine fecundity applying the simple linear regression equation $\mathrm{F}=\mathrm{a}+\mathrm{bx}$, where $\mathrm{F}=$ fecundity, $\mathrm{x}=$ total length, total body weight or ovarian weight, $a$ is the intercept and $b$ the rate of increase in fecundity in relation to the variables in the study. The mean absolute fecundity was estimated in 68280 oocytes which corresponds to an average LT of $21.3 \mathrm{~cm}$ (range: 10749 to 134665 oocytes in specimens with sizes varying from 18.5 to $28.0 \mathrm{~cm}$ ). The best correlation based on regression analysis between the number of oocytes with LT, PT and PGon was with the latter, where the equation was $\mathrm{N} .^{\circ}$ of oocytes $=1618.1 \mathrm{PGon}+11735\left(\mathrm{r}^{2}=0.7917 ; \mathrm{p}<0.05\right)$.

Key words: silver mylossoma, Mylossoma duriventre, fecundity, Amazon fisheries, Ucayali

\section{INTRODUCCIÓN}

La Amazonia presenta una gran diversidad de recursos ícticos, habiéndose identificado más de 800 especies en el Perú (Ortega et al., 2011). Entre la gran diversidad de peces utilizados para consumo se encuentra la palometa (Mylossoma duriventre), disponible en los mercados locales en los estados fresco-refrigerado, seco y salado (Riofrío et al., 2008).

La palometa es una especie omnívora (Carolsfeld et al., 2003; Prieto, 2006), que se caracteriza por tener el cuerpo fuertemente comprimido, discoide, abdomen aquillado y sin espina predorsal. Ocasionalmente presenta una mancha oscura sobre el opérculo. La coloración del cuerpo es ceniza plateada, con aletas hialinas, a excepción de la aleta anal, que posee una tonalidad anaranjada y es densamente escamada. Posee de 17 a 19 radios dorsales, 34 a 37 radios anales y 18 a 22 sierras detrás de la aleta ventral que la diferencia de Mylossoma aureum, especie más parecida y que presenta de 13 a 15 radios dorsales, 28 a 34 radios anales y 10 a 15 sierras detrás de la aleta ventral. Es un desovador total (Mota-Soares et al., 2008). Es importante como pez de consumo y su captura en la pesca comercial se realiza principalmente con redes de cerco, conocidas como honderas, y en menor proporción por redes de arrastre y cortinas (IMARPE, 2008).
Los trabajos de investigación llevados a cabo estuvieron enfocados a la época de reproducción, talla de primera madurez, volúmenes de desembarques, esfuerzo de pesca y lugares más importantes de captura, resaltando las subcuencas de Tamaya, Sheshea e Iparia en Ucayali (IMARPE, 2012). La estimación de la cantidad de ovocitos que una hembra podría expulsar en un desove (considerado como fecundidad), es uno de los indicadores más utilizados para evaluar el potencial reproductivo de una especie y sus posibilidades para la piscicultura (Vazzoler, 1996). Asimismo, se emplea para calcular la supervivencia desde el huevo hasta el reclutamiento y el tamaño de la población a partir de la estimación de la producción anual de huevos (Baxter, 1963). No obstante la importancia de la fecundidad, este parámetro no ha sido estimado, por lo que el presente trabajo se abocó a estimar la fecundidad de la especie en el periodo reproductivo 20112012.

\section{Materiales y Métodos}

El estudio se realizó en la zona de Pucallpa, localizada en el centro oriente del Perú, como parte de los estudios de investigación desarrollados en la amazonia por la Universidad Nacional Mayor de San Marcos (UNMSM) y el Instituto del Mar del Perú (IMARPE) sobre las principales especies 
Cuadro 1. Parámetros corporales de la palometa (Mylossoma duriventre) en la época reproductiva (Pucallpa, Perú) $(\mathrm{n}=49)$

\begin{tabular}{lcc}
\hline Variable & $\begin{array}{c}\text { Promedio } \\
\pm \text { d.e. }\end{array}$ & Rango \\
\hline $\begin{array}{l}\text { Longitud total } \\
(\mathrm{cm})\end{array}$ & $21.3 \pm 2.0$ & $18.5-28.0$ \\
$\begin{array}{l}\text { Peso total } \\
(\mathrm{g})\end{array}$ & $255.5 \pm 78.2$ & $134-544$ \\
$\begin{array}{l}\text { Peso de gónada } \\
(\mathrm{g})\end{array}$ & $34.9 \pm 13.7$ & $6.5-72.0$ \\
\hline
\end{tabular}

Cuadro 2. Número de ovocitos y su relación con parámetros corporales en palometa (Mylossoma duriventre) durante la época reproductiva (Pucallpa, Perú)

\begin{tabular}{lc}
\hline Variable & Promedio \pm d.e. \\
\hline Número de ovocitos $(\mathrm{n})$ & $62,280 \pm 24,968$ \\
Ovocitos $(\mathrm{n})$ por $\mathrm{LT}^{1}(\mathrm{~cm})$ & $3,163 \pm 1,023$ \\
Ovocitos $(\mathrm{n})$ por $\mathrm{PT}^{2}(\mathrm{~g})$ & $268 \pm 81$ \\
Ovocitos $(\mathrm{n})$ por $\mathrm{PG}^{3}(\mathrm{~g})$ & $1,976 \pm 301$ \\
\hline${ }^{1}$ Longitud total & \\
${ }^{2}$ Peso total & \\
${ }^{3}$ Peso de gónada &
\end{tabular}

explotadas por la pesca comercial. Se trabajó con ejemplares de palometa (Mylossoma duriventre), procedentes de las subcuencas de Tamaya, Iparia, Genepanshea y Aruya, dentro del departamento de Ucayali, y que fueron parte de los desembarques de pescado en el puerto de Pucallpa entre octubre de 2011 y enero de 2012. La información de las estadísticas de pesca fue tomada entre las 05:30 y 07:00 horas de lunes a sábado. En cada desembarque se adquirían entre $10 \mathrm{y}$ $20 \mathrm{~kg}$ de material biológico que era trasladado de inmediato al laboratorio.
Se registró la longitud total (LT) mediante un ictiómetro graduado al $0.5 \mathrm{~cm}$. La LT considera la longitud del pez desde el extremo del hocico hasta la terminación de la aleta caudal. Asimismo, se registró el peso total (PT) en una balanza de $2 \mathrm{~kg}$ con precisión de $0.1 \mathrm{~g}$ y el peso de las gónadas (Pgón) en una balanza de $200 \mathrm{~g}$ con sensibilidad de $0.01 \mathrm{~g}$. Se anotaron el sexo y el estadio de madurez, aplicando la escala de seis estadios para hembras, empleada por Riofrío (2002) en boquichico (Prochilodus nigricans), donde se consideran los estadios I = inmaduro, II = en reposo, $\mathrm{III}=$ en maduración inicial, $\mathrm{IV}=$ en maduración avanzada, $\mathrm{V}=$ desove, y VI $=$ desovado.

En el presente estudio solo se emplearon ejemplares con presencia de ovocitos; es decir, hembras en estadios IV y V, llegando a obtenerse únicamente 49 ejemplares. Se tomaron submuestras de las gónadas y fueron preservadas en solución de formol al 5\% y, posteriormente, se realizó el conteo manual de los ovocitos (FAO, 1965). El resultado del conteo en la submuestra se extrapoló al peso total de las gónadas de cada ejemplar. Con la información del número de ovocitos, LT, PT y Pgón, se realizaron los diagramas de dispersión y se observó el tipo de relación entre las variables. De acuerdo a ello, se aplicó la regresión lineal Fecundidad $=\mathrm{a}+\mathrm{bx}$, donde «a» es el intercepto, «X» la variable de interés $\mathrm{y}$ «b» el coeficiente de regresión que indica el incremento de la fecundidad, según Vazzoler (1982). Las regresiones y significancia estadística $(\alpha=0.05)$ de los coeficientes de correlación se probaron con el paquete MINITAB v. 16.

\section{Resultados y Discusión}

Las medidas de LT, PT y Pgón se muestran en el Cuadro 1. El número estimado total de ovocitos y su relación con estas variables se presentan en el Cuadro 2. 
(a)

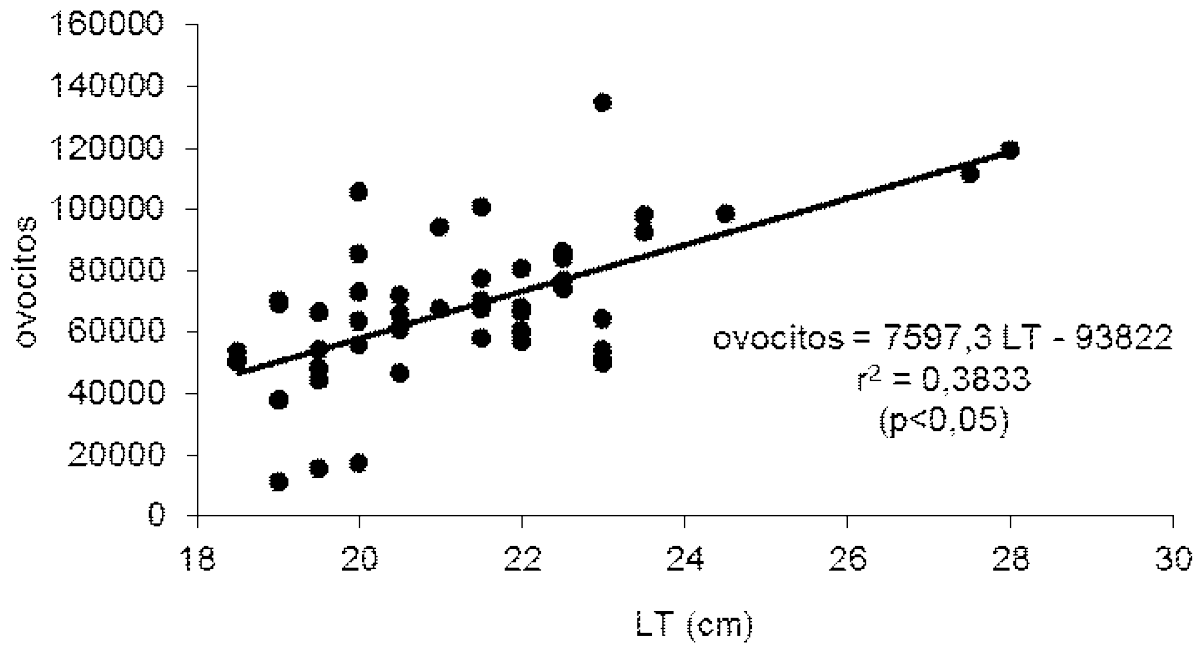

(b)

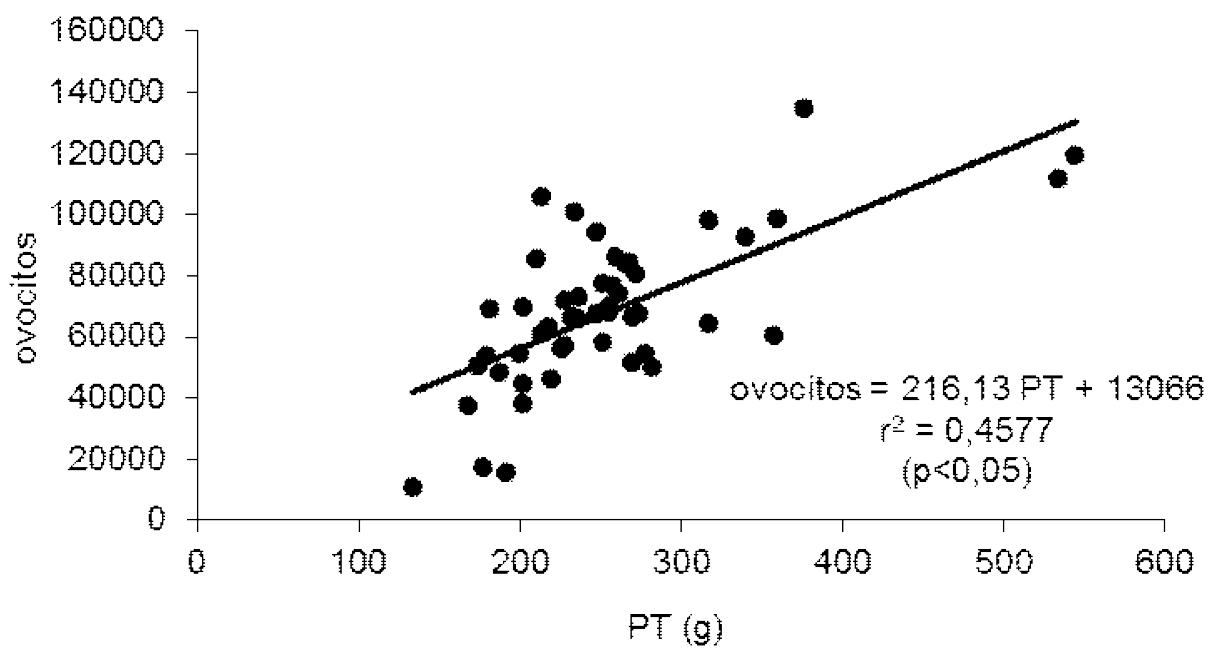

(c)

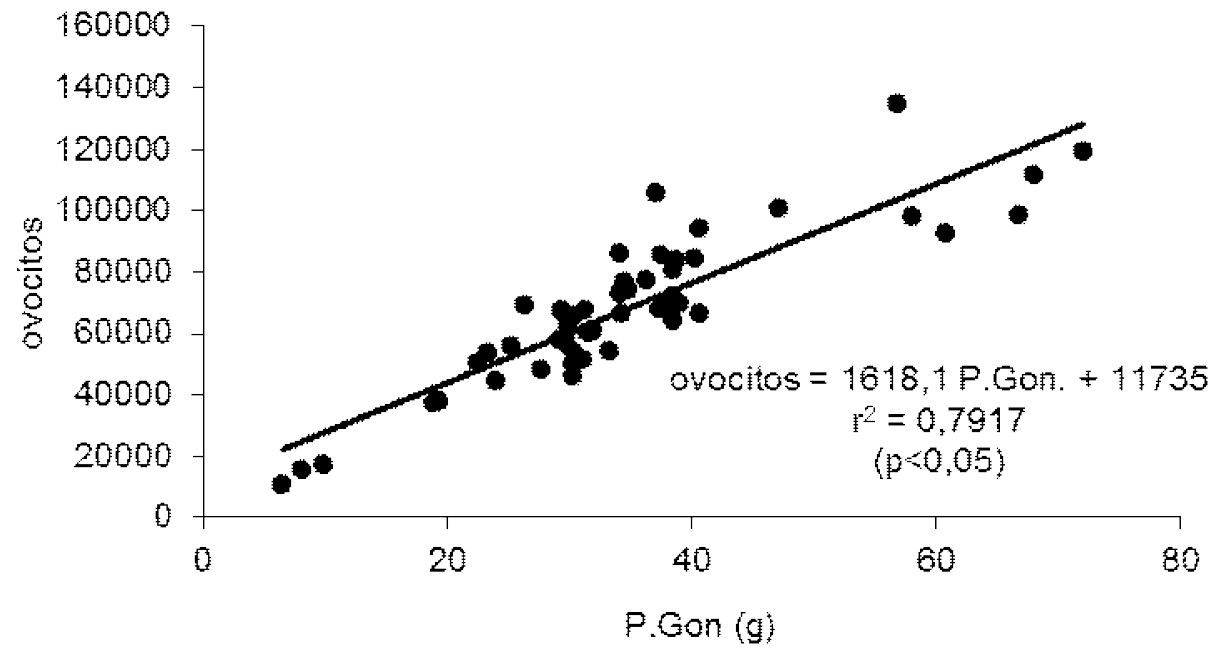

Figura 1. Fecundidad de palometa considerando a) Longitud total, b) Peso total y c) Peso de la gónada 
Se observa una mayor dispersión de la fecundidad cuando se relaciona con la LT (32\%) y el PT (30\%), pero es menor cuando se relaciona con el PGón (15\%) (Fig. 1). El mayor coeficiente de regresión se observa con el peso de la gónada. Useche et al. (1993) reportan para la especie en el río Cafre (Orinoquia, Colombia) una fecundidad promedio de 124885 huevos (rango: 65368 a 253641 ) en 10 ejemplares de $26.2 \mathrm{~cm}$ de longitud estándar (LE) promedio (medida entre el extremo del hocico y la unión de los huesos hipurales). Estos autores citan a Lugo (1989) quien reporta una fecundidad promedio de 129128 huevos (rango: 30996 a 293 940) en ejemplares del río Tomo (Vichada, Colombia). La fecundidad encontrada en el presente estudio es relativamente menor a estos reportes, considerando que se ha trabajado con tallas entre 18.5 y $28.0 \mathrm{~cm}$ de LT.

\section{Literatura Citada}

1. Baxter IG. 1963. A comparison of fecundities of early and late maturity stages of herring in the north-western North Sea. Conseil Permanent International pour l'Exploration de la Mer 154: $170-174$

2. Carolsfeld J, Harvey B, Ross C, Baer A. 2003. Migratory fishes of South America: biology, fisheries and conservation status. Canada: IDRC. 372 p.

3. FAO. 1965. Manual in fisheries sciences N. ${ }^{\circ}$ 1. Manual of methods in fisheries biology. Rome: Food and Agriculture Organization of the United Nations. 53 p.

4. [IMARPE] Instituto del Mar del Perú. 2008. Informe técnico final del proyecto «Monitoreo de la pesquería comercial en Pucallpa y Yarinacocha durante el año 2008 (Ucayali-Perú)». Lima: IMARPE. $51 \mathrm{p}$.

5. [IMARPE] Instituto del Mar del Perú. 2012. Informe técnico final del proyec to «Monitoreo de la pesquería comercial en Pucallpa durante el año 2012 (Ucayali-Perú)». Lima: IMARPE. 32 p.

6. Mota-Soares MG, Costa EL, SiqueiraSouza FK, Beltrão dos Anjos HD, Yamamoto KC, Carvalho Freitas $C$. 2008. Peixes de lagos do Médio Rio Solimões. $2^{\mathrm{a}}$ ed. Manaus, Brazil: Instituto I-Piatam. $160 \mathrm{p}$.

7. Ortega H, Hidalgo M, Correa E, Espino J, Chocano L, Trevejo G, Meza $V$, et al. 2011. Lista anotada de los peces de las aguas continentales del Perú. Lima: MINAM/MHN-UNMSM. 48 p.

8. Prieto QE. 2006. Caracterización de la pesquería en las lagunas de Yahuarcaca (Amazonas, Colombia) y pautas para su manejo sostenible. Tesis de Magíster. Venezuela: Univ Nacional Experimental de los llanos de Venezuela Ezequiel Zamora. $113 \mathrm{p}$.

9. Riofrío JC. 2002. Aspectos biométricos y reproductivos de boquichico Prochilodus nigricans Agassiz, 1829 (Pisces: Prochilodintidae) en Ucayali, Perú. Rev Peru Biol 9: 111-115. doi: 10.15381/ rpb.v9i2.2529

10. Riofrío J, Ferré R, Velarde D. 2008. Contribución para el manejo de la pesquería comercial en Pucallpa (Ucayali, Perú). En: Pinedo D, Soria C (eds). El manejo de las pesquerías en los ríos tropicales de Sudamérica. Bogotá: CIID/ IDRC. 237 p.

11. Useche C, Cala P, Hurtado H. 1993. Sobre la ecología de Brycon siebenthalae y Mylossoma duriventris (Piscis: Characidae) en el río Cafre, Orinoquia. Caldasia 17:341-352.

12. Vazzoler AE. 1982. Manual de métodos para estudos biológicos de populações de peixes. Reprodução e crescimento. Sao Paulo, Brasil: CNPq. $106 \mathrm{p}$.

13. Vazzoler AE. 1996. Biología de reprodução de peixes teleósteos: teoria y prática. Maringá, PR: Ed Nupelia. 169 p. 